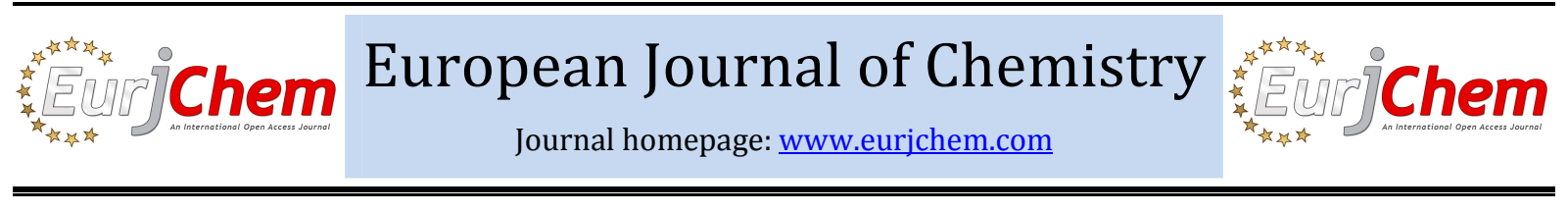

\title{
Chemical constituents and cytotoxic activities of the extracts of Melilotus indicus
}

\author{
Sayed Abdel Kader Ahmed a and Mahmoud Al-Refai b,* \\ a Department of Chemistry, Faculty of Science, Beni-Suef University, Beni-Suef, 62514, Egypt \\ b Department of Chemistry, Faculty of Science, Al Al-Bayt University, Mafraq, 25113, Jordan \\ *Corresponding author at: Department of Chemistry, Faculty of Science, Al Al-Bayt University, Mafraq, 25113, Jordan. \\ Tel.: +962.2.629700.2141. Fax: +962.2.6297021. E-mail address: mahmoudalrefai@aabu.edu.jo (M. Alrefai).
}

\section{ARTICLE INFORMATION}

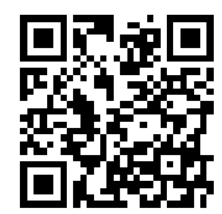

DOI: $10.5155 /$ eurjchem.5.3.503-506.1070

Received: 07 April 2014

Received in revised form: 10 May 2014

Accepted: 13 May 2014

Online: 30 September 2014

\section{KEYWORDS}

Fabaceae

Phenolics

Flavonoids

Melilotus indicus

Cytotoxic activity

Phytochemical studies

\section{Introduction}

The family leguminosae is one of the largest families of flowering plants, consisting of about 463 genera and 18000 species [1]. Melilotus is a genus of plants including 20-25 species that are widely distributed all over the world. Melilotus indicus (M. indicus) belongs to the family Leguminosae (Fabaceae). It is an annual or biennial herb from 10 to $50 \mathrm{~cm}$ in height (rarely to one metre), with yellow flowers native to northern Africa, Europe and Asia, but naturalized throughout the rest of the world [2].

Flavonoids have received an increasing attention in the last decade, especially for their potential protective effects against degenerative diseases linked to oxidative stress [3-6]. Chemoprevention is a rapidly growing practical approach that focuses on cancer prevention by the administration of one or more synthetic or naturally occurring agents to suppress or reverse the process of carcinogenesis. It is becoming increasingly clear that chemopreventive compounds present in diet offer great potential in the fight against cancer by inhibiting the process of carcinogenesis through regulation of cell-defensive and cell death machineries [7]. Dietary chemopreventive substances are regarded as being generally safe, inexpensive and they have been found to contain various phytochemicals which are antioxidant in nature [7]. In Egypt, the leaf extract of $M$. indicus have been utilized as food additives for the prevention of many chronic diseases. The Melilotus species have been found to contain coumarins (Coumarin, dihydrocoumarin and coumarol), acids (o-Hydroxycinnamic, melilotic, coumarinic and o-coumaric), coumarin glycosides, fatty acids (Linoleic acid) and flavonoids (Quercetin and coumestrol), triterpenes (Melilotoside A2 and adzukisaponin V) as well as xylan [8,9] Some of Melilotus species have been found to possess antioxidant and antibacterial properties $[10,11]$. The aim of the work described herein was to isolate and identify the flavonoid constituents of M. indicus, and to evaluate the cytotoxicity of its extracts.

\section{Experimental}

\subsection{Instrumentation}

${ }^{1} \mathrm{H}$ and ${ }^{13} \mathrm{C}$ NMR spectra were registered in a Bruker Advance DRX-400 apparatus. Chemical shifts $(\delta)$ are reported in ppm and coupling constants (J) are reported in Hz. Mass spectra were registered in a Finnigan MAT90 spectrometer operating with ionization energy of $159 \mathrm{eV}$, at positive and negative modes. IR and UV-Vis spectra were registered in the spectrophotometers Shimadzu IR-408, in $\mathrm{KBr}$ discs, and Shimadzu UV-160A, respectively. Melting points were determined in a MQAPF-301 apparatus. 

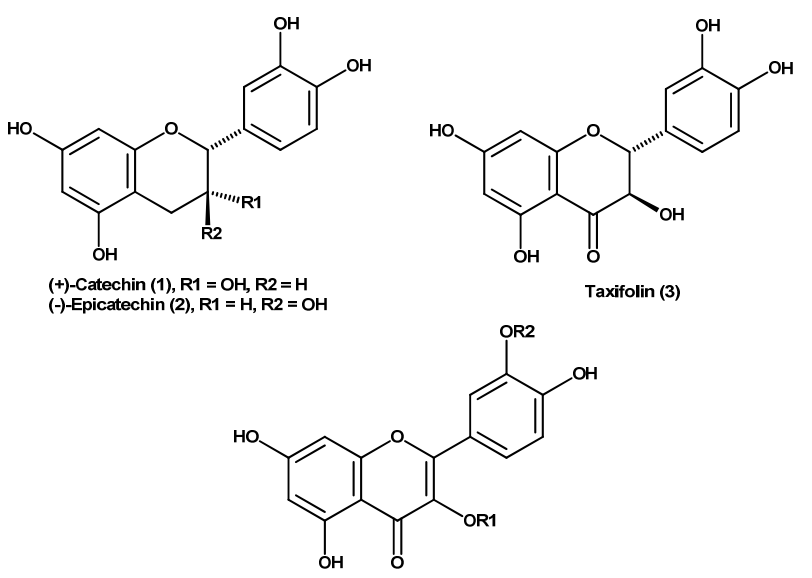

Quercetin (4), R1 = H, R2 = H

Quercetin 3-O- $\beta$-D-glucopyranoside (5), R1 $=\beta$-D-glucopyranoside, R2 = H Quercetin 3-O- $\alpha$-L-rhamnopyranoside (6), R1 $=\alpha-L$-rhamnopyranoside, $R 2=H$

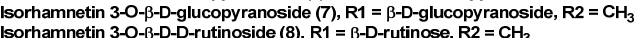

Figure 1. Structures of the isolated flavonoids.

\subsection{Plant material}

The aerial parts of $M$. indicus were collected from the Eastern Nile region, Beni-Suef city on March 2013. Identification of the plant was confirmed by the Department of Flora, Agricultural Museum, Ministry of Agriculture and Herbarium of the Department of Botany, Faculty of Science, Cairo University. Voucher specimen was kept in Herbarium, National Research Center, Giza, Egypt.

\subsection{Extraction and isolation}

The air dried and powdered aerial parts of $M$. indicus $(1.8$ $\mathrm{kg}$ ) were macerated in ethanolic solution $(70 \%)$. The solvent was removed under reduced pressure, and the residue was dissolved in hot water and extracted with chloroform, ethyl acetate and, $n$-butanol, successively. The $n$-butanol extract ( 47 g), a dark brown gum, was subjected to vacuum liquid chromatography (VLC) on silica gel using a gradient elution system of $n$-hexane:EtOAc (10:0, 9:1, 8:2, 7:3, 6:4, 5:5, 4:6, 3:7, $2: 8,1: 9$ and $0: 10 ; v: v)$. Twenty-five fractions were obtained and spotted on TLC using the same solvent system. Similar TLC profiles were grouped to yield 8 fractions (S1-S8). Fraction S3 was chromatographed on a polyamide column eluted with water followed by water/methanol solutions with increasing contents of methanol $(20-70 \%)$ and finishing with methanol. The collected fractions $(217,25 \mathrm{~mL}$ each) were combined according to their TLC analysis. Fractions 14-17 (1.3 g; eluted with water:methanol $(4: 1, v: v)$ were dissolved in methanol and subjected to a sephadex LH-20 column and eluted with methanol for further purification to afford compounds 1 (43 $\mathrm{mg}$ ) and $\mathbf{2}$ (36 mg). Compound 4 (28 $\mathrm{mg}$ ) was purified from S5 on a silica gel column using a mobile phase of hexane:ethyl acetate $(1: 1, v: v)$. The fraction S6 was chromatographed over a silica gel column with $\mathrm{CHCl}_{3}: \mathrm{MeOH}(30: 1, v: v)$ as an eluent to give seven sub-fractions $\left(\mathrm{S}_{1}{ }_{1}-\mathrm{S} 1_{7}\right)$. The sub-fraction $\mathrm{S} 1_{3}$ was subjected to a Sephadex LH-20 (methylene chloride:MeOH, 1:1, $v: v$ ) and purified with silica gel prep. HPLC (Econosil囚 RP-18, $10 \mu$ column, $250 \times 22 \mathrm{~mm}, 47 \% \mathrm{MeOH}$ ) to yield compounds 3 (39 mg). The sub-fractions $\mathrm{S}_{1}$ and $\mathrm{S}_{1} 6$ was also subjected to a Sephadex LH-20 (methylene chloride:MeOH, 1:1, v:v) and purified with silica gel prep. HPLC (Apollo Silica $5 \mu$ column, $250 \times 22 \mathrm{~mm} ; n$-hexane:CHCl $3: E t 0 A c, 7: 3: 1, v: v: v)$ to yield compounds 5 (22 mg) and 6 (31 mg). Fraction S8 were further TLC chromatographed on silica gel eluted with EtOAc:MeOH:
$\mathrm{H}_{2} \mathrm{O}(10: 1: 1, v: v: v)$ to obtain compounds $7(27 \mathrm{mg})$ and 8 (20 $\mathrm{mg}$ ) (Figure 1).

(+)-Catechin: Color: Off-white amorphous powder. M.p.: 208-211 ${ }^{\circ} \mathrm{C} .{ }^{1} \mathrm{H}$ NMR $\left(400 \mathrm{MHz}, \mathrm{CD}_{3} \mathrm{OD}, \delta, \mathrm{ppm}\right): 2.62(\mathrm{dd}, 1 \mathrm{H}$, $J=16.1$ and $8.1 \mathrm{~Hz}, \mathrm{H}-4 \mathrm{ax}), 2.93(\mathrm{dd}, 1 \mathrm{H}, J=16.1$ and $5.4 \mathrm{~Hz}, \mathrm{H}-$ 4 eq $), 3.98(\mathrm{~m}, 1 \mathrm{H}, \mathrm{H}-3), 4.64(\mathrm{~d}, 1 \mathrm{H}, J=7.5 \mathrm{~Hz}, \mathrm{H}-2), 5.87(\mathrm{~d}, 1 \mathrm{H}$, $J=2.3 \mathrm{~Hz}, \mathrm{H}-6), 5.94$ (d, 1H, $J=2.3 \mathrm{~Hz}, \mathrm{H}-8), 6.73(\mathrm{dd}, 1 \mathrm{H}, J=8.1$ and $\left.2.0 \mathrm{~Hz}, \mathrm{H}-6^{\prime}\right), 6.81\left(\mathrm{~d}, 1 \mathrm{H}, J=8.1 \mathrm{~Hz}, \mathrm{H}-5^{\prime}\right), 6.89(\mathrm{~d}, 1 \mathrm{H}, J=$ $2.0 \mathrm{~Hz}, \mathrm{H}-2$ '). ${ }^{13} \mathrm{C}$ NMR (100 MHz, $\left.\mathrm{CD}_{3} \mathrm{OD}, \delta, \mathrm{ppm}\right): 83.2$ (C-2), 69.1 (C-3), 28.8 (C-4), 157.9 (C-5), 96.7 (C-6), 158.1 (C-7), 95.9 (C-8), 157.3 (C-9), 101.2 (C-10), 132.5 (C-1'), 115.6 (C-2'), 146.3 (C-3'), 146.5 (C-4'), 116.5 (C-5'), 120.4 (C-6'). ESI-MS $(\mathrm{m} / \mathrm{z}): 291.0[\mathrm{M}+\mathrm{H}]^{+} .[\alpha]_{\mathrm{D}}=+8.0\left(\mathrm{CH}_{3} \mathrm{OH}, c 0.23\right)$.

(-)-Epicatechin: Color: Colorless amorphous powder. M.p.: 175-176 ${ }^{\circ} \mathrm{C} .{ }^{1} \mathrm{H}$ NMR $\left(400 \mathrm{MHz}, \mathrm{CD}_{3} \mathrm{OD}, \delta, \mathrm{ppm}\right): 2.74(\mathrm{dd}, 1 \mathrm{H}$, $J=16.8$ and $2.9 \mathrm{~Hz}, \mathrm{H}-4 \mathrm{ax}), 2.86(\mathrm{dd}, 1 \mathrm{H}, J=16.8$ and $4.3 \mathrm{~Hz}, \mathrm{H}-$ 4eq), 4.19 (br. s, 1H, H-3), 4.80 (br. s, $1 \mathrm{H}, \mathrm{H}-2$ ), 5.92 (d, 1H, J = $2.3 \mathrm{~Hz}, \mathrm{H}-8$ ), 5.95 (d, $1 \mathrm{H}, J=2.3 \mathrm{~Hz}, \mathrm{H}-6), 6.76$ (d, $1 \mathrm{H}, J=8.2, \mathrm{H}-$ 5'), 6.80 (dd, $1 \mathrm{H}, J=8.2$ and $\left.1.8 \mathrm{~Hz}, \mathrm{H}-6^{\prime}\right), 6.98(\mathrm{~d}, 1 \mathrm{H}, J=1.8 \mathrm{~Hz}$, $\mathrm{H}-2$ '). ${ }^{13} \mathrm{C}$ NMR (100 MHz, $\left.\mathrm{CD}_{3} \mathrm{OD}, \delta, \mathrm{ppm}\right): 80.0$ (C-2), 67.6 (C3), 29.4 (C-4), 157.8 (C-5), 96.6 (C-6), 158.1 (C-7), 96.1 (C-8), 157.5 (C-9), 100.3 (C-10), 132.5 (C-1'), 115.5 (C-2'), 146.1 (C3'), 145.9 (C-4'), 116.1 (C-5'), 119.6 (C-6'). ESI-MS (m/z): 291.0 $[\mathrm{M}+\mathrm{H}]^{+} .[\alpha]_{\mathrm{D}}=-29.6\left(\mathrm{CH}_{3} \mathrm{OH}, c 0.23\right)$.

Taxifolin: Color: Yellow crystals. M.p.: $242^{\circ} \mathrm{C} .{ }^{1} \mathrm{H}$ NMR $(600$ $\left.\mathrm{MHz}, \mathrm{CD}_{3} \mathrm{OD}, \delta, \mathrm{ppm}\right): 4.50(\mathrm{~d}, 1 \mathrm{H}, J=11.0 \mathrm{~Hz}, \mathrm{H}-3), 4.92(\mathrm{~d}, 1 \mathrm{H}$, $J=11.0 \mathrm{~Hz}, \mathrm{H}-2), 5.89$ (d, 1H, $J=2.0 \mathrm{~Hz}, \mathrm{H}-8), 5.91(\mathrm{~d}, 1 \mathrm{H}, J=2.0$ $\mathrm{Hz}, \mathrm{H}-6), 6.81\left(\mathrm{~d}, 1 \mathrm{H}, J=8.0 \mathrm{~Hz}, \mathrm{H}-5^{\prime}\right), 6.86$ (dd, $1 \mathrm{H}, J=2.0$ and $\left.8.0 \mathrm{~Hz}, \mathrm{H}-6^{\prime}\right), 6.97$ (d, 1H, J = 2.0 Hz, H-2'). ${ }^{13} \mathrm{C}$ NMR (100 MHz, $\left.\mathrm{CD}_{3} \mathrm{OD}, \delta, \mathrm{ppm}\right): 85.1$ (C-2), 73.6 (C-3), 198.4 (C-4), 164.3 (C-5), 97.3 (C-6), 168.7 (C-7), 96.3 (C-8), 164.5 (C-9), 101.8 (C-10), 129.8 (C-1'), 115.9 (C-2'), 116.1 (C-5'), 147.1 (C-4'), 146.3 (C3'), $120.9\left(\mathrm{C}-6^{\prime}\right)$. FAB-MS $(\mathrm{m} / \mathrm{z}): 305[\mathrm{M}+\mathrm{H}]^{+} .[\alpha]_{\mathrm{D}}=+17.6$ $\left(\mathrm{CH}_{3} \mathrm{OH}, c\right.$ 1.0).

Quercetin: Color: Yellow amorphous powder. M.p.:311-317 ${ }^{\circ} \mathrm{C} .{ }^{1} \mathrm{H}$ NMR $\left(400 \mathrm{MHz}, \mathrm{DMSO}-d_{6}, \delta, \mathrm{ppm}\right): 6.20(\mathrm{~d}, 1 \mathrm{H}, J=1.8 \mathrm{~Hz}$ $\mathrm{H}-6$ ), 6.42 (d, 1H, $J=1.8 \mathrm{~Hz}, \mathrm{H}-8$ ), 6.90 (d, 1H, $J=8.4 \mathrm{~Hz}, \mathrm{H}-5^{\prime}$ ), $7.57\left(\mathrm{~d}, 1 \mathrm{H}, J=2.1 \mathrm{~Hz}\right.$, and $\left.8.4 \mathrm{~Hz}, \mathrm{H}-6^{\prime}\right), 7.69(\mathrm{~d}, 1 \mathrm{H}, J=2.1 \mathrm{~Hz}$, H-2'), 9.33 (s, 2H, OH-3',4'), 9.56 (s, 1H, OH-3), 10.77 (s, 1H, OH7), $12.50(\mathrm{~s}, 1 \mathrm{H}, \mathrm{OH}-5) .{ }^{13} \mathrm{C}$ NMR (100 MHz, DMSO- $\left.d_{6}, \delta, \mathrm{ppm}\right)$ : 147.5 (C-2), 136.4(C-3), 176.6 (C-4), 161.4(C-5), 98.9 (C-6), 164.6 (C-7), 94.1 (C-8), 156.8 (C-9), 103.7 (C-10), 122.7 (C-1'), 116.3 (C-2'), 145.8 (C-3'), 148.4 (C-4'), 115.8 (C-5'), 120.7 (C6'). ESI-MS (m/z): 301.2 [M-H]-. 
Table 1. Cytotoxicity $\left(\mathrm{IC}_{50}\right.$ in $\mu \mathrm{g} / \mathrm{mL}$ )of the extracts of $M$. indicus a.

\begin{tabular}{|c|c|c|c|c|c|c|}
\hline \multirow[t]{2}{*}{ Extract } & \multicolumn{6}{|c|}{$\mathrm{IC}_{50}(\mu \mathrm{g} / \mathrm{mL})$} \\
\hline & MCF-7 & Hep-2 & HepG-2 & HeLa & HCT-116 & Caco-2 \\
\hline Chloroform extract & $22.52 \pm 1.32$ & $25.47 \pm 2.1$ & $9.21 \pm 0.92$ & $15.52 \pm 1.597$ & $11.24 \pm 1.44$ & $13.83 \pm 1.71$ \\
\hline Ethylacetate extract & $42.53 \pm 0.47$ & $17.44 \pm 1.20$ & $34.81 \pm 3.88$ & $41.898 \pm 2.860$ & $21.58 \pm 1.83$ & $26.14 \pm 1.56$ \\
\hline Methanolic extract & $25.65 \pm 1.90$ & $10.86 \pm 1.15$ & $18.79 \pm 1.55$ & $25.41 \pm 0.88$ & $19.36 \pm 1.96$ & $29.14 \pm 1.10$ \\
\hline Doxorubicin $(\mu \mathrm{M})$ & $0.48 \pm 0.04$ & $0.41 \pm 0.09$ & $0.67 \pm 0.07$ & $1.84 \pm 0.19$ & $1.32 \pm 0.11$ & $0.32 \pm 0.03$ \\
\hline
\end{tabular}

a The data shown are mean \pm SD obtained from three independent experiments.

Quercetin 3-O- $\beta$-D-glucopyranoside: Color: Yellow amorphous powder. M.p.: $224-226{ }^{\circ} \mathrm{C} .{ }^{1} \mathrm{H}$ NMR $\left(400 \mathrm{MHz}\right.$, DMSO- $d_{6}$, $\delta$, ppm): 3.28-3.65 (m, 10H, Glc), 5.37 (d, $\left.1 \mathrm{H}, J=7.63 \mathrm{~Hz}, \mathrm{H}-1^{\prime \prime}\right)$, $6.20(\mathrm{~d}, 1 \mathrm{H}, J=1.8 \mathrm{~Hz}, \mathrm{H}-6), 6.40$ (d, $1 \mathrm{H}, J=1.8 \mathrm{~Hz}, \mathrm{H}-8), 6.82$ (d, $\left.1 \mathrm{H}, J=8.62 \mathrm{~Hz}, \mathrm{H}-5^{\prime}\right), 7.53\left(\mathrm{~d}, 1 \mathrm{H}, J=2.12 \mathrm{~Hz}, \mathrm{H}-2^{\prime}\right), 7.67$ (dd, $1 \mathrm{H}, J=2.12$ and $\left.J=8.62 \mathrm{~Hz}, \mathrm{H}-6^{\prime}\right), 9.14\left(\mathrm{~s}, 1 \mathrm{H}, \mathrm{OH}-4^{\prime}\right), 9.65(\mathrm{~s}$, $\left.1 \mathrm{H}, \mathrm{OH}-3^{\prime}\right), 10.81(\mathrm{~s}, 1 \mathrm{H}, \mathrm{OH}-7), 12.60(\mathrm{~s}, 1 \mathrm{H}, \mathrm{OH}-5) .{ }^{13} \mathrm{C}$ NMR (100 MHz, DMSO- $d_{6}, \delta, \mathrm{ppm}$ ): 156.8 (C-2), 133.6 (C-3), 177.5 (C4), 161.6 (C-5), 98.9 (C-6), 164.6 (C-7), 93.8 (C-8), 156.6 (C-9), 104.0 (C-10), 121.6 (C-1'), 115.8 (C-2'), 145.8 (C-3'), 148.8 (C$\left.4^{\prime}\right), 116.2\left(\mathrm{C}^{\prime} 5^{\prime}\right), 122.0\left(\mathrm{C}-6^{\prime}\right), 101.2\left(\mathrm{C}-1^{\prime \prime}\right), 71.6\left(\mathrm{C}-\mathrm{C}^{\prime \prime}\right), 74.4$ (C3"), 70.0 (C-4"), 77.7 (C-5"), 61.5 (C-6"). EI-MS (m/z): 462.9 [M-H]:

Quercetin 3-O- $\alpha$-L-rhamnopyranoside: Color: Yellow amorphous powder. M.p.: $174-179^{\circ} \mathrm{C} .{ }^{1} \mathrm{H}$ NMR $\left(400 \mathrm{MHz}, \mathrm{CD}_{3} \mathrm{OD}, \delta\right.$, ppm): 1.03 (d, $\left.3 \mathrm{H}, J=6.15 \mathrm{~Hz}, \mathrm{CH}_{3} \mathrm{Rha}\right), 3.16-3.94(\mathrm{~m}, 4 \mathrm{H}$, Rha), 5.29 (d, $1 \mathrm{H}, J=1.41 \mathrm{~Hz}, \mathrm{H}-1$ "), 6.23 (d, $1 \mathrm{H}, J=2.0 \mathrm{~Hz}, \mathrm{H}-6)$, $6.42(\mathrm{~d}, 1 \mathrm{H}, J=2.0 \mathrm{~Hz}, \mathrm{H}-8), 6.90$ (d, $\left.1 \mathrm{H}, J=8.5 \mathrm{~Hz}, \mathrm{H}-5^{\prime}\right), 7.28$ (dd, $1 \mathrm{H}, J=2.0$ and $\left.J=8.5 \mathrm{~Hz}, \mathrm{H}-6^{\prime}\right), 7.33\left(\mathrm{~d}, 1 \mathrm{H}, J=2.0 \mathrm{~Hz}, \mathrm{H}-2^{\prime}\right.$ ). ${ }^{13} \mathrm{C}$ NMR (100 MHz, DMSO- $\left.d_{6}, \delta, \mathrm{ppm}\right): 157.34$ (C-2), 134.27(C3), $177.79(\mathrm{C}-4), 161.35$ (C-5), 98.78 (C-6), 164.34 (C-7), 93.70 (C-8), 156.86(C-9), 104.16(C-10), 121.17 (C-1'), 115.52 (C-2'), 145.25 (C-3'), 148.50 (C-4'), 115.72 (C-5'), 120.80 (C-6'), 101.87 (C-1"), 70.43 (C-2"), 70.63 (C-3"), 71.25 (C-4"), 70.11 (C-5"), 17.54 (C-6"). ESI-MS (m/z): 447.1[M-H].

Isorhamnetin 3-O- $\beta$-D-glucopyranoside; 5,7-dihydroxy-2-(4hydroxy-3-methoxy-phenyl)-3-(3,4,5-trihydroxy-6-hydroxy methyl-tetrahydro-pyrane-2-yloxy)-chromen-4-one: Color: Yellow amorphous powder. M.p.: $267-269^{\circ} \mathrm{C} .{ }^{1} \mathrm{H}$ NMR $(400$ $\left.\mathrm{MHz}, \mathrm{CD}_{3} \mathrm{OD}, \delta, \mathrm{ppm}\right): 3.07-3.83$ (m, 6H, Glc), 3.90 (s, 3H, $\mathrm{OCH}_{3}-$ 3'), 5.40 (d, $\left.1 \mathrm{H}, J=8.5 \mathrm{~Hz}, \mathrm{H}-1^{\prime \prime}\right), 6.20$ (d, $1 \mathrm{H}, J=2.0 \mathrm{~Hz}, \mathrm{H}-6$ ), $6.39(\mathrm{~d}, 1 \mathrm{H}, J=1.8 \mathrm{~Hz}, \mathrm{H}-8), 7.05$ (d, 1H, $J=8.4 \mathrm{~Hz}, \mathrm{H}-5$ '), 7.57 (d, $1 \mathrm{H}, J=2.0 \mathrm{~Hz}, \mathrm{H}-2^{\prime}$ ), 7.69 (dd, $1 \mathrm{H}, J=1.8$ and $8.4 \mathrm{~Hz}, \mathrm{H}-6$ '). ${ }^{13} \mathrm{C}$ NMR $\left(100 \mathrm{MHz}\right.$, DMSO- $\left.d_{6}, \delta, \mathrm{ppm}\right): 156.2$ (C-2), 133.0 (C-3), 177.5 (C-4), 161.1 (C-5), 98.8 (C-6), 164.0 (C-7), 93.8 (C-8), 156.4 (C-9), 104.2 (C-10), 121.7 (C-1'), 111.5 (C-2'), 149.9 (C3'), 146.4 (C-4'), 115.6 (C-5'), 122.3 (C-6'), 55.7 (OCH3-3'), 101.0 (C-1"), 74.2 (C-2"), 77.2 (C-3"), 70.0 (C-4"), 76.6 (C-5"), $61.2\left(\mathrm{C}-6^{\prime \prime}\right)$. ESI-MS $(\mathrm{m} / \mathrm{z}): 479[\mathrm{M}+\mathrm{H}]^{+}$.

Isorhamnetin 3-O- $\beta$-D-rutinoside; 5,7-dihydroxy-2-(4hydroxy-3-methoxy-phenyl)-3-[3,4,5-trihydroxy-6-(3,4,5-tri hydroxy-6-methyl-tetrahydropyran-2-yloxymethyl)-tetrahydropyran-2-yloxy]-chromen-4-one: Color: Yellow amorphous powder. M.p.: $186-187{ }^{\circ} \mathrm{C} .{ }^{1} \mathrm{H}$ NMR $\left(400 \mathrm{MHz}, \mathrm{DMSO}-d_{6}, \delta\right.$, ppm): 0.98 (d, $1 \mathrm{H}, J=5.6 \mathrm{~Hz}$, Rha-H-6), 3.07-3.40 (m, 16H, RutH), 3.83 (s, 3H, $\left.\mathrm{OCH}_{3}-3^{\prime}\right), 4.41$ (d, $1 \mathrm{H}, J=10.8 \mathrm{~Hz}$, Rha- $\left.\mathrm{H}-1\right), 5.44$ (d, $1 \mathrm{H}, J=7.3 \mathrm{~Hz}, \mathrm{Glc}-\mathrm{H}-1), 6.19$ (d, $1 \mathrm{H}, J=1.9 \mathrm{~Hz}, \mathrm{H}-6), 6.41$ (d, $1 \mathrm{H}, J=1.8 \mathrm{~Hz}, \mathrm{H}-8), 6.92\left(\mathrm{~d}, 1 \mathrm{H}, J=8.4 \mathrm{~Hz}, \mathrm{H}-5^{\prime}\right), 7.53(\mathrm{dd}, 1 \mathrm{H}, J$ $\left.=2.0,8.4 \mathrm{~Hz}, \mathrm{H}-6^{\prime}\right), 7.86\left(\mathrm{~d}, 1 \mathrm{H}, J=2.0 \mathrm{~Hz}, \mathrm{H}-2^{\prime}\right), 9.83(\mathrm{~s}, 1 \mathrm{H}, \mathrm{OH}-$ 4'), $10.89(\mathrm{~s}, 1 \mathrm{H}, \mathrm{OH}-7), 12.50(\mathrm{~s}, 1 \mathrm{H}, \mathrm{OH}-5) .{ }^{13} \mathrm{C}$ NMR (100 MHz, DMSO- $\left.d_{6}, \delta, \mathrm{ppm}\right): 156.7$ (C-2), 133.2 (C-3), 177.4 (C-4), 161.4 (C-5), 99.1 (C-6), 165.0 (C-7), 94.1 (C-8), 156.5 (C-9), 103.9 (C10), 121.2 (C-1'), 113.5 (C-2'), 149.6 (C-3'), 55.9 (OCH3-3'), 147.1 (C-4'), 115.5 (C-5'), 122.5 (C-6'), 101.5 (glc-C-1), 74.5 (glc-C-2), 76.6 (glc-C-3), 70.8 (glc-C-4), 76.1 (glc-C-5), 67.0 (glcC-6), 101.1 (rha-C-1), 70.5 (rha-C-2), 70.8 (rha-C-3), 72.0 (rhaC-4), 68.5 (rha-C-5), 17.8 (rha-C-6). ESI-MS (m/z): 623 [M-H]; $647[\mathrm{M}+\mathrm{Na}]^{+}$.

\subsection{Cell culture}

MCF-7 (breast adenocarcinoma), Hep-2 (human epithelial laryngeal carcinoma), HepG-2 (hepatocellular carcinoma), HeLa (human cervix adenocarcinoma), HCT-116 (colorectal adenocarcinoma) and Caco-2 (colon adenocarcinoma) cells were obtained from American Type Culture Collection (ATCC). HeLa and Hep-2 cells were cultured in Dulbecco's modified essential medium, and the other cells were maintained in a RPMI-1640 medium in a humidified $5 \% \mathrm{CO}_{2}$ atmosphere at $37^{\circ} \mathrm{C}$. All media were supplemented with penicillin $(100 \mathrm{U} / \mathrm{mL})$, streptomycin $(100 \mu \mathrm{g} / \mathrm{mL})$ and $10 \%$ heat-inactivated fetal bovine serum (FBS).

\subsection{Cytotoxicity assay}

Cytotoxic activities were measured in-vitro the human cell lines with the MTT (3-(4,5-dimethylthiazol-2-yl)2,5-diphenyl tetrazolium bromide) assay [12]. Briefly, a limited number of human cancer cells (5000/well) were seeded onto a 96-well microplate and became attached to the bottom of the well overnight. On the second day of the procedure, the original medium was removed and $200 \mu \mathrm{L}$ new medium containing the tested extracts was added. After an incubation period of $24 \mathrm{~h}$, the living cells were assayed by the addition of $20 \mu \mathrm{L} 5 \mathrm{mg} / \mathrm{mL}$ MTT solution. MTT was converted by intact mitochondrial reductase and precipitated as blue crystals during a $4 \mathrm{~h}$ contact period. The medium was then removed and the precipitated crystals were dissolved in $100 \mu \mathrm{L}$ DMSO during a $60 \mathrm{~min}$ period of shaking. Finally, the reduced MTT was assayed at $545 \mathrm{~nm}$ using a microplate reader, wells with untreated cells being taken as the control. All invitro experiments were carried out on two microplates with at least five parallel wells. Doxorubicin was used as a positive control. Stock solutions of $10 \mathrm{mg} / \mathrm{mL}$ of the tested extracts were prepared with dimethyl sulphoxide (DMSO). The highest DMSO concentration $(0.3 \%)$ of the medium did not have any significant effect on cell proliferation. The dose-response curves of the compounds were fitted by means of the computer program GraphPad Prism 4.0 (Graph Pad Software, San Diego, CA, USA), and IC50 values were calculated.

\section{Results and discussion}

The ethanolic extract of $M$. indicuswas fractionated and purified by various chromatographic techniques. Eight known flavonoids (1-8) were isolated for the first time from this species (Figure 1). The structural elucidation of the isolated flavonoids was achieved through chemical and spectroscopic analysis, including HRMS, UV, ${ }^{1} \mathrm{H},{ }^{13} \mathrm{C}$ and $2 \mathrm{D} \mathrm{NMR}$, and by comparison with reported data. The isolated flavonoids were identified as catechin (1) [13,14], epicatechin (2) [15,16], taxifolin (3) [17,18], quercetin (4) [19-21],quercetin 3-0- $\beta$-Dglucopyranoside (5) [21-23], and quercetin $3-0-\alpha-\mathrm{L}-$ rhamnopyranoside (6) $[21,24-26]$, isorhamnetin 3-0- $\beta$-Dglucopyranoside (7) [21,27-29] and isorhamentin 3-0- $\beta$-Drutinoside $(\mathbf{8})[21,25,30]$. 
The anti-proliferative activity of three extracts from aerial parts of M. indicuswas determined against Hep-2, HepG-2, HeLa, HCT-116 and Caco- 2 cells by MTT assay using doxorubicin as a positive control. The IC $\mathrm{C}_{50}$ values are presented in Table 1.

Phytochemicals have been shown to be effective in preventing malignant transformation of cells in culture and experimentally induced tumorigenesis in various animal models. Mechanistically, chemoprevention with dietary phytochemicals could be achieved by stimulating inactivation of potential carcinogens, inhibition of abnormal cell proliferation, induction of apoptosis and delaying angiogenesis [31]. The present study was undertaken to investigate the potential of $M$. indicus chloroform, ethyl acetate and methanol extract as a chemopreventive agent by evaluating its effect on viable cell number and by evaluating the sensitivity of different cancer cell lines to the extract. The results of the present study demonstrated that, the cytotoxicity of chloroform extracts was superior to that of the methanol and water extracts, it exhibited a dosedependent growth inhibitory effect after a continuous exposure during a $24 \mathrm{~h}$ period. The cytotoxic effect was greater on HepG2 and HCT-116 cell lines. The most effective cytotoxic agent was the chloroform extract against HepG-2, HCT-116, HeLa and Caco-2 cells with an $\mathrm{IC}_{50} 9.21,11.24,15.52$ and $13.83 \mu \mathrm{g} / \mathrm{mL}$, respectively. In contrast, the most potent cytotoxic agent against Hep-2 cells was the methanolic extract with an $\mathrm{IC}_{50}$ $10.86 \mu \mathrm{g} / \mathrm{mL}$.

\section{Conclusion}

Four flavonoid aglycons and four flavonoid glycosides were isolated from ethanolic extract of Melilotusindicus. Their structures were confirmed by comparison of their chromatographic properties, chemical and spectroscopic data (UV, ${ }^{1} \mathrm{H}$ and ${ }^{13} \mathrm{C} \mathrm{NMR)}$ with those reported in the literature. The antiproliferative assay of chloroform, ethyl acetate and methanol extract have been evaluated by MTT assay using doxorubicin as a positive control, and it has been shown that the methanol extract was the most potent antitumor agent against Hep-2 cell line.

\section{Acknowledgements}

We are grateful to Prof. Dr. Ahmed Hafez Elghandour, Professor of organic chemistry, Chemistry Department, Faculty of Science, Beni Suef University for his encouragement and provision of necessary facilities.

\section{References}

[1]. Sprent, J. I. Perspect. Plant Ecol. 1999, 2, 149-162.

[2]. Webb, C. J.; Sykes, W. R.; Garnock-Jones, P. J. Flora of New Zealand. Vol. IV. Naturalised Pteridophytes, Gymnosperms, Dicotyledons. Christchurch, New Zealand, Botany Division, 1988.

[3]. Havsteen, B. H. Pharmacol. Therapeut. 2002, 96, 67-202.

[4]. Martin, S.; Andriantsitohaina, R. Ann. Cardiol. Angeiol. 2002, 51, 304315.

[5]. Noguchi, C.; Niki, E. Free Radical Bio. Med. 2000, 28, 1538-1546.

[6]. Nunez-Selles, A. J. J. Braz. Chem. Soc. 2005, 16, 699-710.

[7]. Syed, D. N.; Afaq, F.; Mukhtar, H. Cancer Biol. 2007, 17, 377-385.

[8]. Khodakov, G. V.; Yu, A.; Akimov, A. S.; Shashkov, P. K.; Kintia, V. I. Adv. Exp. Med. Biol. 1996, 405, 211-222.

[9]. Khodakov, G. V. Chem. Nat. Compd. 2013, 48, 1024-1026.

[10]. Braga, P. C.; Sasso, M. D.; Lattuada, N.; Marabini, L.; Calo, R.; Antonacci, R.; Bertelli, A.; Falchi, M.; Verducci, P. J. Med. Plant Res. 2013, 7, 358365.

[11]. Miri, A.; Rad, J. S.; Alfatemi, S. M. H.; Rad, M. S. Ann. Biol. Res. 2013, 4, 35-41.

[12]. Mosmann, T. J. Immunol. Methods 1983, 65, 55-63.

[13]. Aguiar, R. M.; David, J. P.; David, J. M. Phytochemistry 2005, 66, 23882392.

[14]. Jin, W.; Tu, P. J. Chromatogr. 2005, 1092, 241-245.

[15]. Foo, L. Y.; Newman, R.; Waghorn, G.; Mcnabb, W. C.; Ulyatt, M. J. Phytochemistry 1996, 41, 617-624.

[16]. Chakravarthy, B. K.; Gode, K. D. Planta Med. 1985, 51, 56-59.

[17]. Han, X. H.; Hong, S. S.; Hwang, J. S.; Lee, M. K.; Hwang, B. Y.; Ro, J. S. Arch. Pharm. Res. 2007, 30, 13-17.
[18]. Arriaga, A. M. C.; Castro, M. A. B.; Silveira, E. R.; Braz-Filho, R. J. Braz. Chem. Soc. 2000, 11, 187-190.

[19]. Zheng, Z.; Cheng, K.; Chao, J.; Wu, J.; Wang, M. Food Chem. 2008, 106, 529-535.

[20]. Agrawal, P. K. Carbon-13 NMR of Flavonoids. Elsevier, Amsterdam, 1989.

[21]. Liu, H.; Yan, M.; Jianglin, Z.; Jihua, W.; Ligang, Z.; Mingan, W.; Daoquan W.; Jianguo, H.; Zhu, Y.; Fuyu, Y. Molecules 2010, 15, 7933-7945.

[22]. Harborne, J. B. The flavonoids, Advances in research since 1986 Chapman and Hall, London, 1994.

[23]. Vvedenskaya, I. O.; Rosen, T.; Guido, J. E.; Russell, D. J.; Mills, K. A.; Vorsa, N. J. Agricul. Food Chem. 2004, 52, 188-195.

[24]. Ye, G.; Huang, C. Chem. Nat. Compd. 2006, 42, 232-234.

[25]. Markham, K. R.; Terani, B.; Stanley, R.; Geiger, H.; Mabry, T. J. Tetrahedron 1999, 50, 329-332.

[26]. El-Toumy, S. A.; El-Sharabasy, F. S.; Ghanem, H. Z.; El-Kady, M. U. Kassem, A. F. Aust. J. Basic Appl. Sci. 2011, 5, 1362-1370.

[27]. Markham, K. R. Techniques of flavonoid identification. Academic Press, London, 1982.

[28]. Beck, M. A.; Haberlein, H. Phytochemistry 1999, 50, 329-332.

[29]. D'Agostino, M.; De Simone, F.; Dini, A.; Ramundo, E.; Zollo, F. Phytochemistry 1990, 29, 353-354.

[30]. Wang, J.; Gao, H.; Zhao, J.; Wang, Q.; Zhou, L.; Han, J.; Yu, Z.; Yang, F. Molecules 2010, 15, 5998-6007.

[31]. Kundu, J. K.; Surh, Y. J. Phytochem. Rev. 2009, 8, 333-347. 